\title{
Modern technology and its use as storytelling communication strategy in public health
}

\begin{abstract}
Communication is the best tool to successfully achieve an objective of any intervention focused on solving public health issues. In public health there exist many communication strategies but the most effective is the storytelling communication strategy. Story telling is a way in which message is conveyed to people in the form of narrating stories. The mode of storytelling can be electronic, verbal or printed form. In past decade mode of communication has evolved. Hence the recent development in technology has come up with potential storytelling modes of communication used in public health intervention. Digital storytelling, Virtual games, Photo voice as well as social media applications have provided a platform to both the community and the health practitioners to share health information. Several modes of communication bridge the gap between the practitioner as well as patients, and modern technology is one of it. The paper describes some of the modern storytelling communication strategies used in public health interventions so far. The Internet is the major source of the platform, the modern technology used in storytelling has inherited flaws in it. The mode of storytelling though being effective is not user-friendly to all age groups. Future studies in this field are required to discuss the potential options regarding storytelling interventions. The need of developing optimized communication material in a form which is user-friendly to all age group should be addressed in future research studies.
\end{abstract}

Keywords: communication strategy, storytelling, modern technology, public health, gamification, photo voice
Volume 6 Issue 3 - 2017

\author{
Naiya Patel, Neel Patel \\ Department of Public Health, Long Island University, USA
}

Correspondence: Naiya Patel, Department of Masters in Public Health, Long Island University, Brooklyn, New York, USA Tel 9292807280, Email naiya.patel2014@gmail.com

Received: September II, 2017 | Published: September 18, 2017

\section{Introduction}

The way globalization is skyrocketing in recent years, the need for a common source of information sharing or communicating is rising as well. Moreover, communication in local dialect is important for reaching out to the population of concern. ${ }^{1}$ Interventions which target behavior change amongst the population of concern should be based on thorough understanding of the target population culture as well as ethnicity. ${ }^{2}$ The health message hence can be tailored in a way which makes it culturally relevant and can help us achieve expected outcome. The advances in technology especially, e-technology have resulted into more human socialization. Some people are searching out proactively information related to their well-being via the use of blogs, social networking sites, text messaging, virtual world, etc. More than $80 \%$ of the American population which uses the internet has searched for health information in recent years leaving health professionals with no other choice but to improvise the way of reaching out to the target population and disseminating messages. ${ }^{3}$ People can easily participate with the help of technology. It not only reduces financial cost but also improves quality of participation. These technologies help in the engagement of social network. ${ }^{4}$ More recently, narrative form of communication strategy has surfaced as one of the most promising ways of achieving public health objectives including but not limited to prevention/control of cancer. The narrative form of communication synonymously called by some researchers as Storytelling is an umbrella term including testimonies, literature, journalism, and storytelling with entertaining education. ${ }^{5}$ Storytelling used as communication strategy is proven to be effective and its importance cannot be underestimated. It is one of the oldest ways of communicating and passing on the knowledge as well as skills to the future generation. ${ }^{6}$

There are many platforms through which people nowadays communicate and share their experiences. Similarly, they also seek out help using the same mode of communication tool. There are several ways in which storytelling is disseminated to population; they are as follows: ${ }^{7}$

i. Social networking sites/applications- e.g., Facebook, Twitter.

ii. Mobile phones- e.g., Instagram, Snapchat, Texting, Gamification(games).

iii. Other internet based technologies- e.g., Podcast, Blogs.

Television shows- e.g., Documentaries, Reality shows.

Even though digital storytelling has improved community outreach, there are certain challenges to it like, unclear boundaries, consent, and recruitment to participate, the power of shaping and harm, confidentiality issues as well as the release of material. ${ }^{8}$ The purpose of this paper is to review effective storytelling communication strategy for recent time. It discusses what the current technology tools are used in public health as a source of storytelling communication strategy. The Figure 1 is the survey held by McKinney in 2014. It describes various channels through which interaction occurs between health service provider and patient in Germany, United Kingdom as well as Singapore. The survey helps to estimate the percent frequency per year for several communication channel sources. It was clear that emails and online portals topped the preference over fax and mails, which indicates the frequency of more than four times per year. The 
figure reflects that patients are turning towards the use of modern technology to conventional methods of communication. Moreover, we need to keep in mind that the survey data is not age adjusted. Hence conclusions cannot be generalized regarding ease of preference use like emails, online portals and so on.

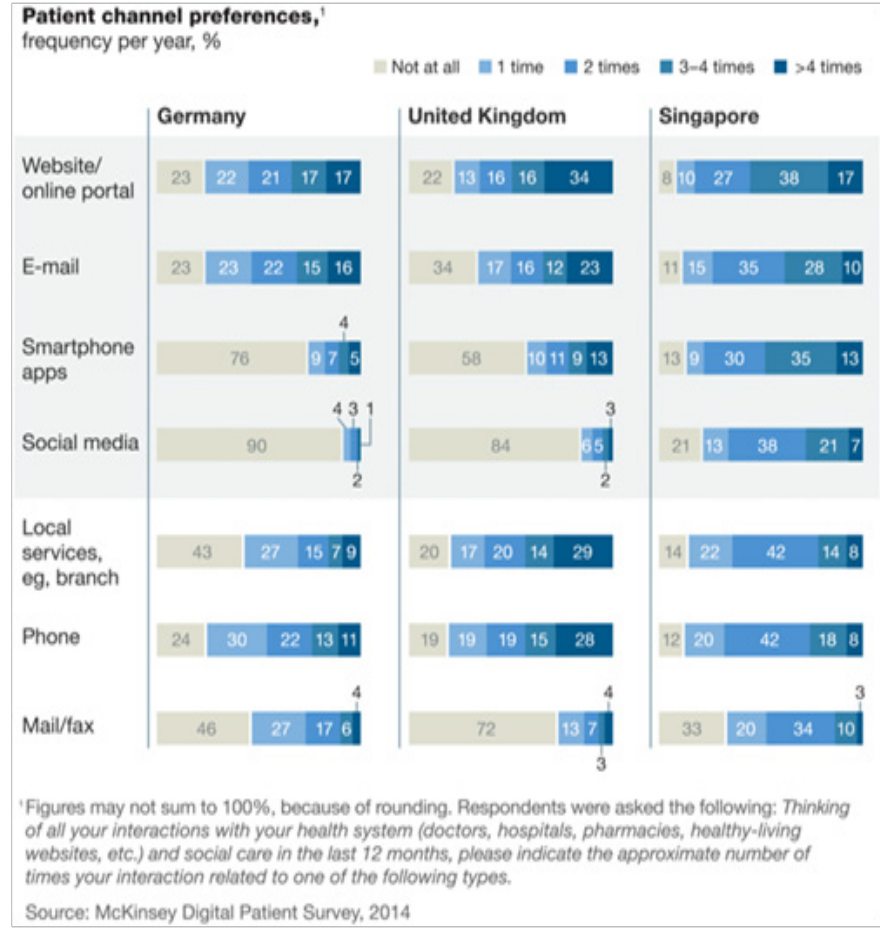

Figure I The Mckinsey digital patient survey in 2014 providing an estimation of percentage frequency per year for interaction with health care provider via several channels.

\section{Types of platform for storytelling}

This section focuses on mobile phones and YouTube channel as one of the most effective modern tools for sharing digitalized storytelling to reach out to people, as well as for being a major platform for seeking information. Mobile phones include gamification, texting, digital storytelling, etc. Digital storytelling provides an opportunity to ordinary people for creating their short autobiographical films. Those autobiographies can be broadcasted on social media as well as telecasted on television. ${ }^{9}$ Digital storytelling encourages participation of participants in a group activity. It helps participants to share their stories and ideas. Resulting digital stories are short, first person visual narratives that synthesize digital images, audio recordings of a first person, told story, music, and text to document personal experiences. ${ }^{10}$ Digital storytelling combines the past methods of storytelling with the recent advances in technology and carries people through the past, present and future. ${ }^{11}$

A. Mobile phone- They are used as a tool for storytelling communication strategy recently, to approach a population of concern. Storytelling has played an effective role since past many years as per. ${ }^{12}$ The combination of mobile and web technology is emerging in the market with the term known as "Community Generated Media"(CGM) and is the most cost effective way of reaching out to people over a wireless network. ${ }^{13}$ The two major ways in which mobile phones used are as follows:
Gamification in apps- Gamification is the use of game design elements in non-game context. It is also observed in the paper that approximately $60 \%$ of health initiatives include game elements and includes rewards and incentives, in combination with expected behaviors to increase motivation and maintain behavior over time. Games play a major role in shaping individuals behavior if game elements are designed accordingly. Some of the behavioral constructs included in the mobile game app are described as follows: ${ }^{14}$

i. Psychological-It includes providing general information, self-monitoring facility to track behavior and stress management by providing information about how to cope stress.

ii. Physical- It includes skills training by providing training for performing a particular behavior and simplicity by providing options to resolve barriers which come in the way of achieving expected behavior

iii. Motivation- It includes sub constructs of automatic and secondly reflective. Automatic, on the one hand, is achieved by providing incentives like gaining points and social support by sharing it with social network group.

iv. Opportunity- It also has two sub constructs named social which includes peer pressure and second is physical which includes cues to action along with stimulus control.

B. Mobile Self-Management system- Mobile phones are considered to be one of the most promising tools in self-management related diseases which include lifestyle related disease because of their availability and increase in usage. The self-management apps to control diabetes are available for free in smart phones and have increased in recent years on a commercial basis as well. Effective medical care comes along with consistent self-management sustained by the patient. For example, the uses of self-management tool helping with self-management of blood glucose and a healthier diet. The major components of this application are the Diabetes diary and its core features:

i. Wireless data which is transmitted automatically from a blood glucose meter and a step counter.

ii. Nutrition/diet routine recording.

iii. Feedback is given by the diary after analysis of raw data.

iv. Goal setting feature which enables person to set goals regarding nutrition habit step counts.

v. Lastly, a general advisory function related to self-management. ${ }^{15}$

C. YouTube channel ${ }^{16}$ described the community based participatory research (CBPR) method known as photo voice had received growing attention in health education and related fields. The community based participatory research provides the opportunity of giving real time feedback by the community itself. YouTube like social platform equips us to reach out to the community most affordably and effectively. YouTube like social software tools facilitate intergenerational interaction as well as enhance co-creativity of older people as discussed in the paper by Harley D. ${ }^{17}$ So, it is one of the most effective tools of storytelling communication strategy used for public health campaigns.

D. Lastly, other effective tools of modern technology are virtual videos, social movements, blog posts, etc. They all fall under the 
wide umbrella of latest technology "Web 2.0". ${ }^{18}$ Hence the advance in the technology itself is a wide umbrella with multiple tools under it which are most effective ways to reach out to people and community for effective public health programs.

The Figure 2 explains about willingness by consumers to use technology for their health-related activities. The most preferred ones were phone calls followed by checking health information, e-mails, scheduling an appointment, etc. Since the data is not age adjusted, future surveys might want to address the age factor, which can help us optimize the insights for real world data.

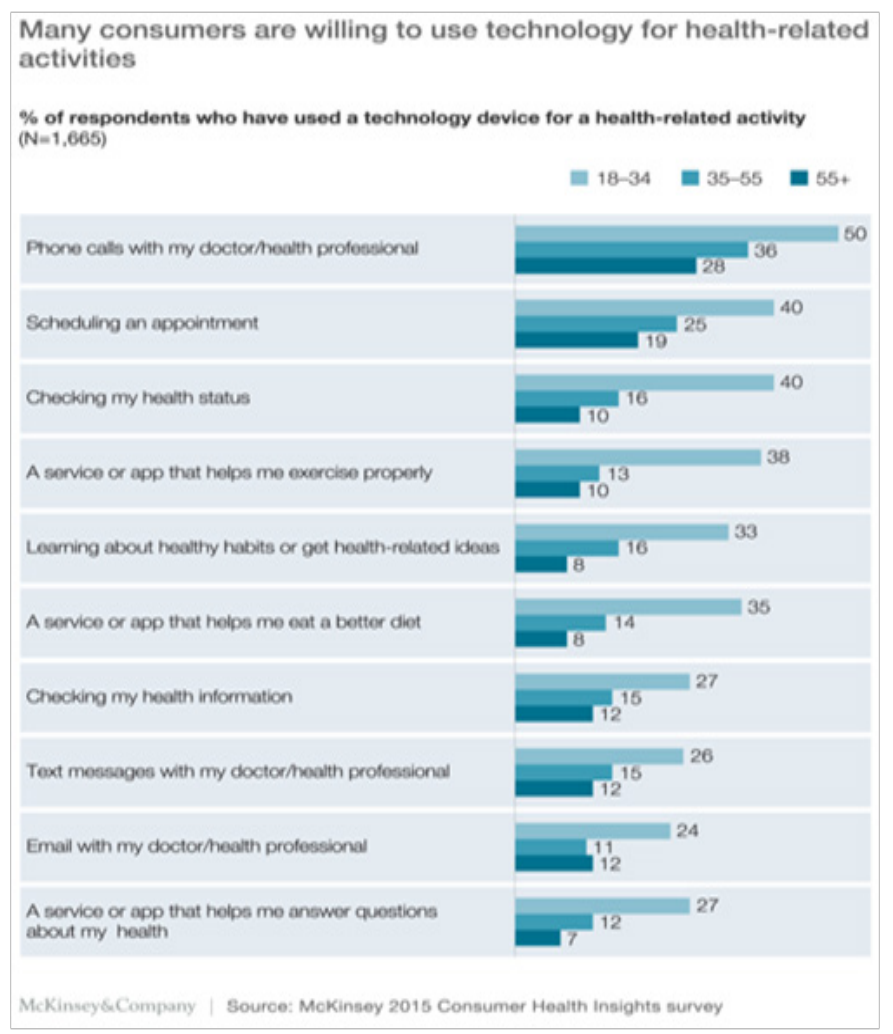

Figure 2 McKinney Consumer Health Insights Survey in 2015 describing several modes of communication used in health related activities by consumers of health services.

\section{Conclusion}

The advances in technology have improved the health care system eventually with some unavoidable challenges like unclear boundaries, recruitment to participate, harm confidentiality and release of material. Moreover, accessibility to latest tools for every kind of population might not be an affordable option. Future research might open the doors for the possibility to the limitations mentioned above. The sources mentioned in the paper are most effective way of reaching to the community, but further studies might want to explore which specific tool is efficient over other after addressing the challenges. Regarding YouTube channels and Facebook, not everyone around the world can access it. The elderly population who are not tech savvy might be unable to share their own stories through these platforms. Additionally, not every part of the globe can provide required resources, like internet services. There are several parts of the world where the population is deprived of electrical connection to their house example, Indian villages. If there is no electricity, people cannot access modern technology sources. In recent times, there are platforms which influence the population accessing it unfairly. The examples are advertisements appealing to our eyes but unhealthy for our body such as sugary drinks, soda, etc. These advertisement brands also use storytelling strategy for selling the products to consumers. Hence future studies need to be focused considering the fact, that till what extent a message is beneficial or harmful to the consumers. We need to set the clear boundaries about any storytelling concept used to shape participant's behavior. The concept of storytelling should be in a positive range over the negative range regarding influencing its viewers. Hence, future researches studies should be focused on certain factors which can help reach to the community in most effective and optimized way. The factors are setting boundaries regarding shaping one's behavior, addressing confidentiality issues of the actors participating in video clips, user-friendly applications/tools for elder age group, providing basic resources to the public regarding using modern technologies- like the internet, etc. Further study in the field should come up with more practical interventions which can be easily used in real world.

\section{Acknowledgments}

Professor Jelena Malogajski, Department of Masters in Public Health, Long Island University, Brooklyn; Professor Bojana Beric, Chair of Department of Public Health, Long Island University.

\section{Conflict of interest}

Author declares that there is no conflict of interest.

\section{References}

1. Mushengyezi A. Rethinking indigenous media: rituals, 'talking'drums and orality as forms of public communication in Uganda. Journal of African Cultural Studies. 2003;16(1):107-117.

2. Larkey LK, Hecht M. A model of effects of narrative as culture-centric health promotion. J Health Commun. 2010;15(2):114-135.

3. Macario E, Ednacot EM, Ullberg L, et al. The changing face and rapid pace of public health communication. $J$ Comm Healthcare. 2011;4(2):145-150.

4. Thackeray R, Hunter M. Empowering youth: Use of technology in advocacy to affect social change. Journal of Computer $\square$ Mediated Communication. 2010;15(4):575-591.

5. Kreuter MW, Green MC, Cappella JN, et al. Narrative communication in cancer prevention and control: a framework to guide research and application. Ann Behav Med. 2007;33(3):221-235.

6. Haigh C, Hardy P. Tell me a story-a conceptual exploration of storytelling in healthcare education. Nurse Educ today. 2011;31(4):408-411.

7. Clar C, Dyakova M, Curtis K, et al. Just telling and selling: current limitations in the use of digital media in public health: a scoping review. Public health. 2014;128(12):1066-1075.

8. Gubrium AC, Hill AL, Flicker S. A situated practice of ethics for participatory visual and digital methods in public health research and practice: A focus on digital storytelling. Am J Public Health. 2014;104(9):1606-1614.

9. Burgess J. Hearing ordinary voices: Cultural studies, vernacular creativity and digital storytelling. Continuum: Journal of Media \& Cultural Studies. 2006;20(2):201-214.

10. DiFulvio GT, Gubrium AC, Fiddian Green A, et al. Digital storytelling as a narrative health promotion process: Evaluation of a pilot study. Int Q community health Educ. 2016;36(3):157-164. 
11. Cueva M, Kuhnley R, Lanier A, et al. Promoting Culturally Respectful Cancer Education Through Digital Storytelling. Int $J$ Indiq Health. 2016;11(1):34-49.

12. Dimond JP, Dye M, LaRose D, et al. Hollaback!: the role of storytelling online in a social movement organization. ACM, USA; 2013. p. $477-490$.

13. Frohlich DM, Rachovides D, Riga K, et al. Story Bank: mobile digital storytelling in a development context. ACM, USA; 2009. p. 1761-1770.

14. Lister C, West JH, Cannon B, et al. Just a fad? Gamification in health and fitness apps. JMIR serious games. 2014;2(2):e9.
15. Tatara N, Arsand E, Skrovseth SO, et al. Long-term engagement with a mobile self-management system for people with type 2 diabetes. JMIR MHealth and UHealth. 2013;1(1):e1.

16. Catalani C, Minkler M. Photovoice: A review of the literature in health and public health. Health Educ Behav. 2010;37(3):424-451.

17. Harley D, Fitzpatrick G. YouTube and intergenerational communication: the case of Geriatric1927. Universal access in the information society. 2009;8(1):5-20.

18. Lefebvre RC. The new technology: the consumer as participant rather than target audience. Social Marketing Quarterly. 2007;13(3):31-42. 